\title{
The Functional Role of Individual- Alpha Based Frontal Asymmetry in the Evaluation of Emotional Pictures: Evidence from Event-Related Potentials
}

\author{
Tao Suo ${ }^{1}$, Lei Liu ${ }^{2 *}$, Chaoyang Chen ${ }^{2}$ and Entao Zhang ${ }^{1}$ \\ ${ }^{1}$ Institute of Psychology and Behavior, Department of Psychology, School of Education, Henan University, Kaifeng, China, \\ ${ }^{2}$ Department of Psychology, Ningbo University, Ningbo, China
}

OPEN ACCESS

Edited by:

Qinghua He,

Southwest University, China

Reviewed by:

Fanchang Kong,

Central China Normal

University, China

Matt R. Judah,

Old Dominion University,

United States

Wenbo Luo,

Liaoning Normal University,

China

*Correspondence:

Lei Liu

liulei@nbu.edu.cn

Specialty section:

This article was submitted

to Psychopathology,

a section of the journal

Frontiers in Psychiatry

Received: 06 July 2017 Accepted: 07 September 2017 Published: 27 September 2017

Citation:

Suo T, Liu L, Chen C and Zhang E (2017) The Functional Role of Individual-Alpha Based Frontal

Asymmetry in the Evaluation of Emotional Pictures: Evidence from

Event-Related Potentials.

Front. Psychiatry 8:180.

doi: 10.3389/fpsyt.2017.00180
The perceptual processing of emotional stimuli is subject to the regulation of brain function. This study investigated whether frontal electroencephalography (EEG) alpha asymmetry at resting conditions predicted the evaluation of emotional picture stimuli by event-related potentials (ERPs). In this study, participants first completed a 2-min resting task, and then passively viewed emotional pictures. The results showed that left active individuals had smaller frontal EEG alpha asymmetry scores to negative pictures than to positive and neutral pictures, whereas right active individuals had similar frontal EEG alpha asymmetry scores to negative, positive, and neutral pictures. Furthermore, the study showed a larger P300 to negative pictures than to positive and neutral pictures for left active individuals; however, there were no significant ERP differences to negative, positive, and neutral pictures for right active individuals. These findings suggest that frontal EEG alpha asymmetry at resting conditions can reflect interindividual differences in emotional perception tendencies to emotional picture stimuli.

Keywords: frontal electroencephalography alpha asymmetry, emotional processing, resting electroencephalography, event-related potential, P300

\section{INTRODUCTION}

The question what factors account for individual differences in the evaluation of emotional stimuli continues to be a central issue in the field of affective neuroscience. Research suggested that emotional processing was regulated by a functional lateralization of frontal cortex (1-5). A reliable correlate of frontal activity is frontal electroencephalography (EEG) alpha asymmetry that is reflected in the alpha frequency band (typically $8-13 \mathrm{~Hz}$ ) measured with electroencephalography (EEG). Recent evidences suggested that there was an inverse relation between activity within the alpha range and cortical processing. For example, when underlying cortical systems was in active processing, the alpha tended to decrease (6-8). Thus, this study examined how frontal EEG alpha asymmetry was related to individual differences in the evaluation of emotional stimuli.

Frontal EEG alpha asymmetry includes two types of asymmetry: frontal EEG alpha asymmetry at resting conditions and frontal EEG alpha asymmetry during emotional challenge. The former is related this asymmetry to various trait-like individual differences and been referred to as trait frontal EEG alpha asymmetry; the latter is investigated in relation to manipulations that intended to influence emotional states and been labeled state frontal EEG alpha asymmetry $(1,2,9)$. 
In line with a stable trait view, several studies suggested that frontal EEG alpha asymmetry in resting conditions can moderate the transient EEG asymmetry response to emotional stimuli. For instance, more left frontal EEG alpha asymmetry at resting conditions had been linked to superior emotional flexibility $(4,10)$, more effective emotion regulation $(11,12)$, lower stress-induced cortisol levels (5) as well as to less negative and more positive affect $(13,14)$. These studies suggest that individual frontal EEG alpha asymmetry at resting conditions can be used to predict the response to emotional stimuli.

Event-related potentials (ERPs) have high temporal resolution and can be used to study the unfolding of emotional processing. Especially, the P300 and late positive potential (LPP) are considered to be good neural indexes to track these stages of emotional processes (15-18). Researchers showed that P300 is sensitive to large number of cognitive processing, such as probability, task difficulty, and resource allocation $(19,20)$. In studies using emotional stimuli, the P300 has been summarized as reflecting "the allocation of capacity-limited resources toward motivationally salient environment stimuli," in which motivationally relevant stimuli (e.g., emotional stimuli) naturally and automatically arouse and direct attentional resources $(21,22)$. Some research showed that emotional stimuli can attract our attention and produce larger P300 amplitude for emotional pictures compared to neutral pictures $(17,21,23)$. Following P300 amplitude, a sustained positivity was observed, which was sensitive to the emotional stimuli. The LPP has been considered as a component related to the subjective evaluation of emotional stimuli. There was larger LPP amplitude for positive and negative pictures than neutral pictures $(21,24)$. However, no studies so far investigated whether frontal asymmetry predicted the evaluation of emotional picture stimuli using ERPs.

This study aimed to examine whether frontal EEG alpha asymmetry at resting conditions predicted the evaluation of emotional picture stimuli by ERPs. For the purposes of this study, we were interested in the P300 and LPP response as they related to processing of emotion stimuli. In this study, participants first completed a 2-min resting task, and then passively viewed emotional pictures. We expected that the transient EEG asymmetry response to emotional picture stimuli would be moderated by frontal EEG alpha asymmetry at resting conditions; we also expected left frontal EEG alpha asymmetry group, not right frontal EEG alpha asymmetry group, would appear different neural responses to emotional picture stimuli in the P300 as well as LPP.

\section{MATERIALS AND METHODS}

\section{Participants}

Thirty-six undergraduates (25 females, 19-32 years old, $\mathrm{M}=22.44$ years, $\mathrm{SD}=2.71$ years) were recruited to participate in this study. All participants were right-handed with normal or corrected-to-normal vision and had no psychological or neurological disorders. All participants gave their written informed consent, and the study was approved by the local Ethics Committee of Henan University.

\section{Stimuli Material}

Emotional pictures were chosen from IAPs based on the ratings of arousal and valence. There were 62 pictures in each of the following three groups: positive pictures, negative pictures, and neutral pictures. In a pilot study, 291 Chinese participants were asked to rate the arousal and valence of all 704 IAPs pictures [see the Method section of Zhang and Zhou $(24,25)]$. In this study, the means and SDs of the arousal and valence ratings for the three picture sets were as follows: negative pictures (arousal: $\mathrm{M}=5.75, \mathrm{SD}=0.45$, valence: $\mathrm{M}=1.99$, $\mathrm{SD}=0.33$ ), positive pictures (arousal: $\mathrm{M}=5.72, \mathrm{SD}=0.45$, valence: $\mathrm{M}=7.25, \mathrm{SD}=0.38$ ), and neutral pictures (arousal: $\mathrm{M}=4.21, \mathrm{SD}=0.27$, valence: $\mathrm{M}=5.10, \mathrm{SD}=0.45$ ) (see the Supplementary Material). One-way ANOVA was examined for valence and arousal, respectively. The main effect of valence was significant, $F(2,122)=2,641.52, p<0.001, \eta_{p}^{2}=0.98$. The post hoc test showed that positive pictures, negative pictures, and neutral pictures were significantly different in valence ( $p$ s $<0.001)$. The main effect of arousal was significant, $F(2$, $122)=321.66, p<0.001, \eta_{p}^{2}=0.84$. The post hoc test showed that emotional pictures differed significantly from neutral pictures in arousal $(p s<0.001)$. However, there was no significant difference between positive pictures and negative pictures in arousal $(p>0.05)$.

\section{Procedure}

After completing Beck depression inventory (BDI) (26), Beck anxiety inventory (BAI) (27), and Positive and Negative Affect Schedule (PANAS) (28), participant was seated in a comfortable chair facing a computer screen at a distance of $70 \mathrm{~cm}$. Electrodes were attached. First, recording of resting EEG was abstained for each participant. Participants were asked to complete a 2-min resting task. Research showed that time of recording on asymmetry scores did not impacted the internal consistency (29), and that time of 2-min recording was similarly reliable to time of 8-min recording for resting EEG asymmetry (30-32). Then, participants were asked to complete the emotion task in which participants passively viewed emotional pictures. Participants viewed 186 items total, which were divided into three blocks based on the picture types (positive pictures, negative pictures, and neutral pictures). There were 62 trials for each block. In each trial, a blank screen was presented for $300 \mathrm{~ms}$, and then emotional picture was presented for 1,000 ms following a randomized interstimulus interval of $2,200-2,700 \mathrm{~ms}$.

\section{EEG Recording and Analysis}

Electroencephalography data were recorded using the NeuroScan recording and analysis system with $40 \mathrm{Ag} / \mathrm{AgCl}$ electrodes positioned according to the International 10-20 system. EEG signals were acquired by a DC model with a sampling rate of $1,000 \mathrm{~Hz}$ and a bandwidth of $100 \mathrm{~Hz}$. By electrodes placed above and below the outer orbits of the left eye, vertical and horizontal electrooculograms (EOGs) were recorded. During EEG recording, the electrode on the left mastoid served as a reference. For all of the electrodes, impedance was kept under $5 \mathrm{k} \Omega$. 


\section{EEG Analysis}

The EEG asymmetry measures were taken from the 2-min resting task, and the asymmetry scores were calculated from both eyes open condition and eyes closed condition. EEG data were re-referenced offline to Cz. All data were inspected visually, to eliminate intervals in which ocular or muscle artifacts occurred. Power spectra were derived by means of a fast Fourier transform with a Hamming window (epoch length $1 \mathrm{~s}, 50 \%$ overlap) for resting task for each participant. For consistency with previous research $(33,34)$, we focused on the alpha band $(8-13 \mathrm{~Hz})$ in the frontal electrodes F3 and F4. A laterality coefficient (LC) indexing relative left versus right sided activation was used. EEG LC were computed as follows: $\mathrm{LC}=((L-R) /(L+R)) \times 100$. Positive values indicated higher alpha activity in the left than in the right hemisphere (4). There was a long tradition for the calculation of LC in laterality research (35). EEG studies showed that there is a perfectly correlation between LC and another common metric $(\ln L-\ln R)(36,37)$.

\section{ERP Analysis}

The ERP measures were taken from the emotion task. ERP averages were locked to the onset of the picture from $100 \mathrm{~ms}$ before picture onset until 1,000 ms after. For each participant, the average number of artifact-free trials of each ERP grand average was 50, and the minimum number was 45 . EEG data were re-referenced offline to the averaged mastoids and were filtered using a $0.05-30 \mathrm{~Hz}$ bandwidth. Vertical and horizontal EOGs were filtered out based on independent components analysis used in ERP studies. The rejection criterion of artifact was amplitude of $\pm 100 \mu \mathrm{V}$. EEG data were averaged by time window from $100 \mathrm{~ms}$ pre-picture cues to $1,000 \mathrm{~ms}$ post-picture cues. Based on previous studies (21-23), there were two time windows for mean amplitude measurements, including the P300 (300-500 ms) and the LPP (500-1,000 ms). The present studies computed the centro-parietal region by averaging across electrodes $(\mathrm{CP} 3, \mathrm{CPz}$, CP4, P3, and Pz). Research showed P300 and LPP were typically observed in centro-parietal electrodes $(21,22,38,39)$.

\section{Data Analysis}

According to a median split of frontal EEG alpha asymmetry scores during the 2-min resting task, individuals can be divided into two groups: left active group vs. right active group. Previous studies showed that this was a valid method for separating the impact of individual differences in frontal EEG alpha asymmetry at resting conditions $(4,40)$. To investigate the influence of frontal EEG alpha asymmetry at resting conditions on picture perception, repeated measures ANOVAs were performed including valence (negative, positive, and neutral) as within subject variable and group (left vs. right) as between subject variable for LC, P300 amplitude, and LPP amplitude, respectively. A Greenhouse-Geisser correction was applied to all ANOVAs when necessary. The significance levels were set at 0.05 .

\section{RESULTS}

Participants were subdivided into groups based on the median of frontal EEG alpha asymmetry scores at F3/F4 electrodes during the 2-min resting task (left active group: $18,-10.64 \pm 8.12$; right active group: $18,9.15 \pm 7.14$ ). The independent samples $t$-test showed that there were no significant differences between left active group and right active group for age, BAI, BDI, PANAS (negative emotion), and PANAS (positive emotion), respectively (see Table 1).

\section{EEG Results}

Figure 1 showed the difference between left active group and right active group on frontal EEG alpha asymmetry scores for the three pictures stimuli.

For the LC, the ANOVA results revealed that there were not significant main effects of valence, $F(2,68)=1.44, p=0.24$, $\eta_{p}^{2}=0.04$, and group, $F(1,34)=0.11, p=0.74, \eta_{p}^{2}<0.01$. However, a significant valence $\times$ group interaction was obtained, $F(2,68)=3.54, p=0.04, \eta_{p}^{2}=0.09$; simple effect analysis suggested that for left active group, negative pictures induced smaller LC than positive $(p=0.02)$ and neutral $(p=0.02)$ pictures, whereas there was no significant difference between neutral pictures and positive pictures; for the right active group, there were not significant differences among negative, positive, and neutral pictures (see Figure 1).

\section{ERP Results}

Figure 2 showed the grand average ERPs during viewing negative, positive, and neutral pictures for left active group and right active group.

TABLE 1 | The description results of age, Beck anxiety inventory (BAI), Beck Depression Inventory (BDI), and Positive and Negative Affect Schedule (PANAS) for left active group and right active group.

\begin{tabular}{lccr}
\hline Types & $\begin{array}{c}\text { Left active } \\
\text { group }(\boldsymbol{N}=\mathbf{1 8})\end{array}$ & $\begin{array}{c}\text { Right active } \\
\text { group }(\boldsymbol{N}=\mathbf{1 8})\end{array}$ & $\boldsymbol{t}(\boldsymbol{p})$ \\
\hline Age & $21.89 \pm 2.54$ & $23.00 \pm 2.83$ & $-1.24(0.22)$ \\
BAl & $31.94 \pm 8.86$ & $29.11 \pm 5.64$ & $1.15(0.26)$ \\
BDI & $13.56 \pm 9.84$ & $12.00 \pm 7.37$ & $0.54(0.60)$ \\
PANAS & $19.39 \pm 7.75$ & $18.61 \pm 7.01$ & $0.32(0.75)$ \\
(negative emotion) & & & \\
PANAS & $22.22 \pm 7.27$ & $23.89 \pm 7.32$ & $-0.69(0.50)$
\end{tabular}

(positive emotion)

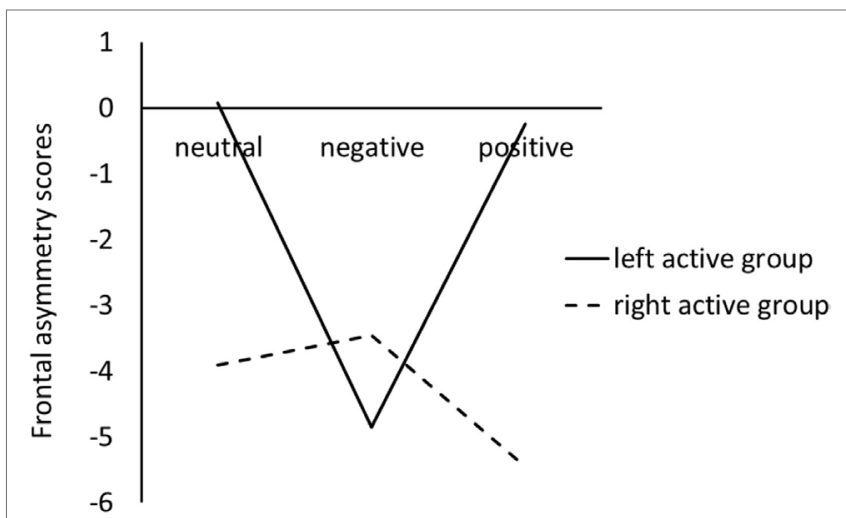

FIGURE 1 | Frontal electroencephalography alpha asymmetry scores during viewing negative, positive, and neutral pictures for left active group and right active group. 
left active group

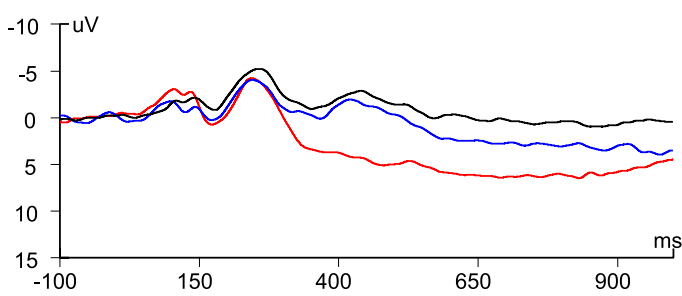

right active group

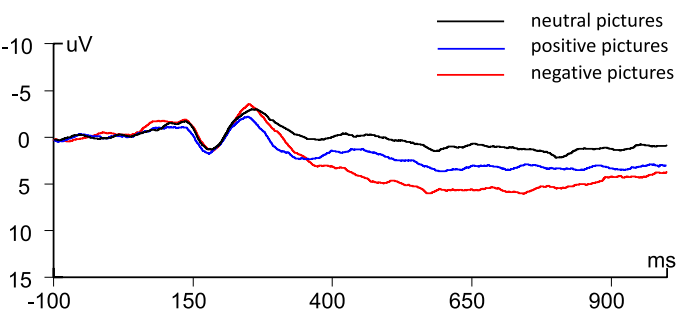

FIGURE 2 | Grand average event-related potentials (ERPs) showing the average across centro-parietal electrodes. Left: ERPs for neutral, negative, and positive images collapsed across left active group. Right: ERPs for right active group.

\section{left active group}

\section{P300(300-500 ms)}

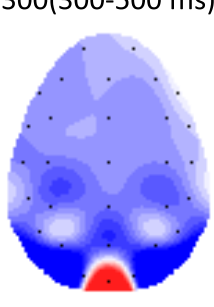

negative - neutral

negative - positive
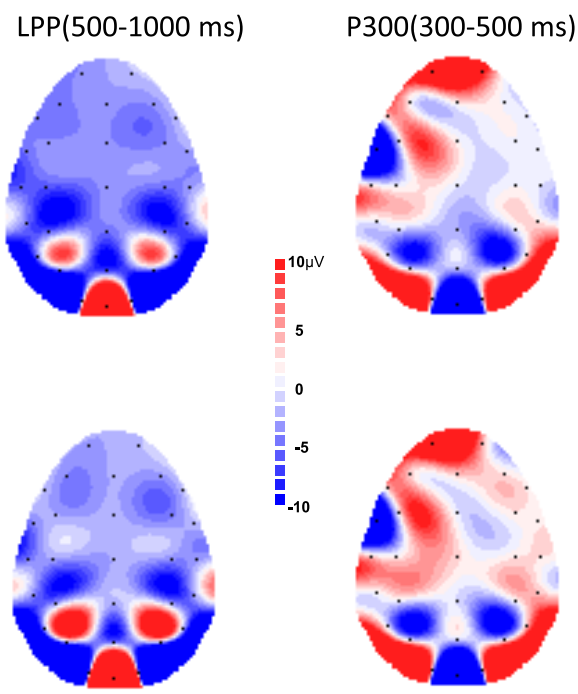

\section{right active group}

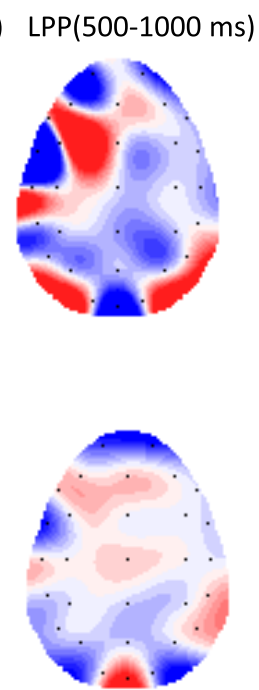

FIGURE 3 | Topographic maps depicting voltage differences for negative minus neutral pictures and negative minus positive pictures in the time range of P300 (300-500 ms) and late positive potential (LPP) (500-1,000 ms) for left active group (left) and right active group (right).

Figure 3 showed voltage differences for negative minus neutral pictures and negative minus positive pictures in the time range of P300 (300-500 ms) and LPP (500-100 ms) for left active group and right active group.

\section{P300}

The ANOVA results revealed a significant main effect of valence, $F(2,68)=17.00, p<0.001, \eta_{p}^{2}=0.33$, indicating negative pictures induced greater P300 amplitude than positive $(p<0.01)$ and neutral $(p<0.001)$ pictures (see Figure 2). There was no significant main effect of group, $F(1,34)=0.08, p=0.78, \eta_{p}^{2}<0.01$. However, a significant valence $\times$ group interaction was also obtained, $F(2,68)=4.67, p=0.02, \eta_{p}^{2}=0.12$. Simple effect analysis suggested that for left active group, negative pictures induced larger P300 amplitude than positive $(p<0.001)$ and neutral $(p<0.001)$ pictures, whereas there was no significant difference between neutral pictures and positive pictures (see Figures 2 and 3); for right active group, there were no significant differences among negative, positive, and neutral pictures.

\section{Late Positive Potential}

The ANOVA results revealed a significant main effect of valence, $F(2,68)=10.95, p<0.001, \eta_{p}^{2}=0.24$, indicating negative pictures induced greater LPP amplitude than positive $(p=0.02)$ and neutral $(p<0.001)$ pictures, and positive pictures induced greater LPP amplitude than neutral pictures $(p=0.02)$ (see Figure 2). There are no significant main effect of group, $F(1,34)=0.41$, $p=0.53, \eta_{p}^{2}=0.01$, and the interaction between group $\times$ valence was not significant, $F(2,68)=1.71, p=0.19, \eta_{p}^{2}=0.05$.

\section{DISCUSSION}

This study showed that frontal EEG alpha asymmetry at resting conditions predicted the evaluation of emotional picture stimuli. Left active individuals had smaller frontal EEG alpha asymmetry scores to view negative than to view positive and neutral pictures, whereas right active individuals had similar frontal EEG alpha asymmetry scores when viewing negative, positive, and neutral pictures. Furthermore, the study showed a larger P300 
to negative pictures than to positive and neutral pictures for left active individuals; however, there were no significant ERP differences to negative, positive, and neutral pictures for right active individuals.

The EEG finding that there was different neural performance between left active individuals and right active individuals was in line with our expectation. That is, frontal EEG alpha asymmetry at resting conditions can regulate the frontal alpha EEG asymmetry response to emotional picture. This was consistent with previous studies. Coan and Allen (41) showed that individuals with left frontal EEG alpha asymmetry at resting conditions reported increased emotional experience and increased the emotional intensity, compared with individuals with right frontal EEG alpha asymmetry. Kline et al. (40) and Papousek et al. $(4,10)$ showed that individuals with left frontal EEG alpha asymmetry at resting conditions exhibited EEG asymmetry changes when experiencing emotional stimulation, whereas individuals with right frontal EEG alpha asymmetry appeared unresponsive to emotional stimulations. Thus, the results showed there was flexible response when viewing emotional pictures for left active individuals, whereas there was inflexible response for right active individuals.

Especially, the ERP findings showed that compared with right active individuals, left active individuals had larger P300 effect to negative pictures than to positive and neutral pictures. Emotional ERP modulations can be explained by the "negativity-bias" effect. Research showed that there were valence ERP effects that indicated greater P300 magnitude to negative pictures than to neutral pictures or positive pictures (15-17, 42). The "negativity-bias" effect emphasized the intrinsic relevance for negative stimuli (43, 44 ) and was considered to response to attention processing (45, 46). Thus, emotional P300 effects may reflect the rapid attention to the threatening stimuli, and then improve individual processing efficiency (47). According to this view, for individuals with left frontal EEG alpha asymmetry, attention can be automatically directed to a threatening stimulus from an evolutionarily adaptive perspective. However, for individuals with right frontal EEG alpha asymmetry, the "negativity-bias" effect disappeared, which indicated that they did not response to a threat and did not adapt the environment quickly.

The neuro-laterality models of affect and psychopathology provided a theoretical basis for differential involvement of left and right frontal cortical regions in emotional processing. The laterality models assume the left and right frontal cortical hemispheres to be differentially involve in processes modulating affective responses to emotional challenges $(3,36)$. It has been

\section{REFERENCES}

1. Davidson RJ. What does the prefrontal cortex "do" in affect: perspectives on frontal EEG asymmetry research. Biol Psychol (2004) 67:219-33. doi:10.1016/j. biopsycho.2004.03.008

2. Coan JA, Allen JJB, McKnight PE. A capability model of individual differences in frontal EEG asymmetry. Biol Psychol (2006) 72:198-207. doi:10.1016/j. biopsycho.2005.10.003

3. Harmon-Jones E, Gable PA, Peterson CK. The role of asymmetric frontal cortical activity in emotion-related phenomena: a review and update. Biol Psychol (2010) 84:451-62. doi:10.1016/j.biopsycho.2009. 08.010 proposed that greater left frontal EEG activity at baseline is associated with greater affective flexibility as compared to asymmetry in favor of the right hemisphere (4). Our findings support the neuro-laterality models of affect and psychopathology. That is, individual frontal EEG alpha asymmetry at resting conditions can predict the response to emotional stimuli.

This study is based on the view that frontal EEG alpha asymmetry at resting conditions reflects a personal trait to react emotionally. The study demonstrates that frontal EEG alpha asymmetry predicts the evaluation of emotional picture stimuli. On the one hand, frontal EEG alpha asymmetry at resting conditions may moderate the transient EEG asymmetry response to emotional picture stimuli. On the other hand, compared to right active individuals, left active individuals put more attentional resources to negative pictures than to positive and neutral pictures.

\section{ETHICS STATEMENT}

This study was carried out in accordance with the recommendations of the local Ethics Committee of Henan University with written informed consent from all participants. All participants gave their written informed consent in accordance with the Declaration of Henan University. The protocol was approved by the local Ethics Committee of Henan University.

\section{AUTHOR CONTRIBUTIONS}

All the authors took part in planning the theoretical and conceptual basis for the study. All the authors took part in critically reviewing and editing the manuscript.

\section{FUNDING}

This study was supported by the National Natural Science Foundation of China (31400908, 31700952), the National Science Foundation for Post-doctoral Scientists of China (2014M551995), the Humanity and Social Science Youth foundation of Ministry of Education of China (16YJC190013) and the Zhejiang Provincial Natural Science Foundation of China (LQ17C090002) and the Philosophy and Social Science Foundation of Henan Province, China (2015BJY007).

\section{SUPPLEMENTARY MATERIAL}

The Supplementary Material for this article can be found online at http://journal.frontiersin.org/article/10.3389/fpsyt.2017.00180/ full\#supplementary-material.

4. PapousekI, ReiserEM, Weber B, FreudenthalerHH, Schulter G. Frontalbrain asymmetry and affective flexibility in an emotional contagion paradigm. Psychophysiology (2012) 49:489-98. doi:10.1111/j.1469-8986.2011.01324.x

5. Quaedflieg CWEM, Meyer T, Smulders FTY, Smeets T. The functional role of individual-alpha based frontal EEG alpha asymmetry in stress responding. Biol Psychol (2015) 104:75-81. doi:10.1016/j.biopsycho.2014.11.014

6. Davidson RJ. Cerebral asymmetry, emotion and affective style. In: Davidson RJ, Hugdahl K, editors. Brain Asymmetry. Cambridge, MA: MIT Press (1995). p. 361-87.

7. Laufs H, Kleinschmidt A, Beyerle A, Eger E, Salek-Haddadi A, Preibisch C, et al. EEG-correlated fMRI of human alpha activity. Neuroimage (2003) 19:1463-76. doi:10.1016/S1053-8119(03)00286-6 
8. Klimesch W, Sauseng P, Hanslmayr S. EEG alpha oscillations: the inhibitiontiming hypothesis. Brain Res Rev (2007) 53:63-88. doi:10.1016/j.brainresrev. 2006.06.003

9. Coan JA, Allen JJB. The state and trait nature of frontal EEG asymmetry in emotion. In: Hugdahl K, Davidson RJ, editors. The Asymmetrical Brain. Cambridge, MA: MIT Press (2003). p. 565-616.

10. Papousek I, Weiss EM, Schulter G, Fink A, Reiser EM, Lackner HK. Prefrontal EEG alpha asymmetry changes while observing disaster happening to other people: cardiac correlates and prediction of emotional impact. Biol Psychol (2014) 103:184-94. doi:10.1016/j.biopsycho.2014. 09.001

11. Jackson DC, Mueller CJ, Dolski I, Dalton KM, Nitschke JB, Urry HL, et al. Now you feel it, now you don't: frontal brain electrical asymmetry and individual differences in emotion regulation. Psychol Sci (2003) 14:612-7. doi:10.1046/j.0956-7976.2003.psci_1473.x

12. Papousek I, Weiss EM, Perchtold CM, Weber H, Assunção VL, Schulter G, et al. The capacity for generating cognitive reappraisals is reflected in asymmetric activation of frontal brain regions. Brain Imaging Behav (2016) 11(2):577-90. doi:10.1007/s11682-016-9537-2

13. Tomarken AJ, Davidson RJ, Wheeler RE, Kinney L. Psychometric properties of resting anterior EEG asymmetry: temporal stability and internal consistency. Psychophysiology (1992) 29:576-92. doi:10.1111/j.1469-8986.1992. tb02034.x

14. Schmidt B, Hanslmayr S. Resting frontal EEG alpha-asymmetry predicts the evaluation of affective musical stimuli. Neurosci Lett (2009) 460:237-40. doi:10.1016/j.neulet.2009.05.068

15. Keil A, Bradley MM, Hauk O, Rockstroh B, Elbert T, Lang PJ. Largescale neural correlates of affective picture processing. Psychophysiology (2002) 39:641-9. doi:10.1111/1469-8986.3950641

16. Eimera M, Holmes A. Event-related brain potential correlates of emotional face processing. Neuropsychologia (2007) 45:15-31. doi:10.1016/j. neuropsychologia.2006.04.022

17. Olofsson JK, Nordin S, Sequeira H, Polich J. Affective picture processing: an integrative review of ERP findings. Biol Psychol (2008) 77:247-65. doi:10.1016/j.biopsycho.2007.11.006

18. Schacht A, Sommer W. Emotions in word and face processing: early and late cortical responses. Brain Cogn (2009) 69:538-50. doi:10.1016/j. bandc.2008.11.005

19. Polich J. Neuropsychology of P300. In: Luck SJ, Kappenman ES, editors. Handbook of Event-Related Potential Components. Oxford: Oxford University Press (2011). p. 159-88.

20. Luck SJ. An Introduction to the Event-Related Potential Technique. 2nd ed. Cambridge: MIT Press (2014).

21. Hajcak G, MacNamara A, Olvet DM. Event-related potentials, emotion, and emotion regulation: an integrative review. Dev Neuropsychol (2010) 35:129-55. doi:10.1080/87565640903526504

22. Eddy MD, Brunyé TT, Tower-Richardi S, Mahoney CR, Taylor HA. The effect of a brief mindfulness induction on processing of emotional images: an ERP study. Front Psychol (2015) 6:1391. doi:10.3389/fpsyg.2015. 01391

23. Mini A, Palomba D, Angrilli A, Bravi S. Emotional information processing and visual evoked brain potentials. Percept Mot Skills (1996) 83:143-52. doi:10.2466/pms.1996.83.1.143

24. Moser E, Hajcak G, Bukay E, Simons RF. Intentional modulation of emotional responding to unpleasant pictures: an ERP study. Psychophysiology (2006) 43:292-6. doi:10.1111/j.1469-8986.2006.00402.x

25. Zhang J, Zhou RL. Individual differences in automatic emotion regulation affect the asymmetry of the LPP component. PLoS One (2014) 9:e88261. doi:10.1371/journal.pone.0088261

26. Zhang YX, Wang Y, Qian MY. Reliability and validity of beck depression inventory (BDI) examined in Chinese samples. Chin Ment Health J (1990) 4:164-8.

27. Zheng RJ, Hang ZR, Hang JJ, Zhuang XQ, Wang DB, Zheng SY, et al. A study of psychometric properties, normative scores and factors structure of beck anxiety inventory Chinese version. Chin J Clin Psychol (2002) 10:4-6. doi:10.16128/j.cnki.1005-3611.2002.01.002

28. Zhang WD, Diao J, Schick CJ. The cross-cultural measurement of positive and negative affect examining the dimensionality of PANAS. Psychol Sci (2004) 27:77-9. doi:10.16719/j.cnki.1671-6981.2004.01.020
29. Allen JJB, Coan JA, Nazarian M. Issues and assumptions on the road from raw signals to metrics of frontal EEG asymmetry in emotion. Biol Psychol (2004) 6:183-218. doi:10.1016/j.biopsycho.2004.03.007

30. Coan JA, Allen JJ, Harmon-Jones E. Voluntary facial expression and hemispheric asymmetry over the frontal cortex. Psychophysiology (2001) 38:912-25. doi:10.1111/1469-8986.3860912

31. Grouiller F, Vercueil L, Krainik A, Segebarth C, Kahane P, David O. A comparative study of different artefact removal algorithms for EEG signals acquired during functional MRI. Neuroimage (2007) 38:124-37. doi:10.1016/j. neuroimage.2007.07.025

32. Hammer EM, Halder S, Blankertz B, Sannelli C, Dickhaus T, Kleih S, et al. Psychological predictors of SMR-BCI performance. Biol Psychol (2012) 89:80-6. doi:10.1016/j.biopsycho.2011.09.006

33. Lee TW, Yu YWY, Hong CJ, Tsai SJ, Wu HC, Chen TJ. The influence of serotonin transporter polymorphisms on cortical activity: a resting EEG study. BMC Neurosci (2011) 12:33. doi:10.1186/1471-2202-12-33

34. Matsuda I, Nittono H, Allen JJ. Detection of concealed information by P3 and frontal EEG asymmetry. Neurosci Lett (2013) 537:55-9. doi:10.1016/j. neulet.2013.01.029

35. Porac C, Coren S. Lateral Preferences and Human Behaviour. New York: Springer-Verlag (1981).

36. Davidson RJ. Affective style and affective disorders: perspectives from affective neuroscience. Cogn Emotion (1998) 12:307-30. doi:10.1080/026999398379628

37. Papousek I, Schulter G. Covariations of EEG asymmetries and emotional states indicate that activity at frontopolar locations is particularly affected by state factors. Psychophysiology (2002) 39:350-60. doi:10.1017/ S0048577201393083

38. Dunning JP, Hajcak G. See no evil: directing visual attention within unpleasant images modulates the electrocortical response. Psychophysiology (2009) 46:28-33. doi:10.1111/j.1469-8986.2008.00723.x

39. Hajcak G, MacNamara A, Foti D, Ferri J, Keil A. The dynamic allocation of attention to emotion: simultaneous and independent evidence from the late positive potential and steady state visual evoked potentials. Biol Psychol (2013) 92:447-55. doi:10.1016/j.biopsycho.2011.11.012

40. Kline JP, Blackhart GC, Williams WC. Anterior EEG asymmetries and opponent process theory. Int J Psychophysiol (2007) 63:302-7. doi:10.1016/j. ijpsycho.2006.12.003

41. Coan JA, Allen JJB. Varieties of emotional experience during voluntary emotional facial expressions. Ann N Y Acad Sci (2003) 1000:375-9. doi:10.1196/ annals. 1280.034

42. Cuthbert BN, Schupp HT, Bradley MM, Birbaumer N, Lang PJ. Brain potentials in affective picture processing: covariation with autonomic arousal and affective report. Biol Psychol (2000) 52:95-111. doi:10.1016/ S0301-0511(99)00044-7

43. Crawford LE, Cacioppo JT. Learning where to look for danger: integrating affective and spatial information. Psychol Sci (2002) 13:449-53. doi:10.1111/ 1467-9280.00479

44. Öhman A, Mineka S. Fears, phobias, and preparedness: toward an evolved module of fear and fear learning. Psychol Rev (2001) 108:483-522. doi:10.1037/0033-295X.108.3.483

45. Carretie L, Hinojosa JA, Martin-Loeches M, Mercado F, Tapia M. Automatic attention to emotional stimuli: neural correlates. Hum Brain Mapp (2004) 22:290-9. doi:10.1002/hbm.20037

46. Wood S, Kisley MA. The negativity bias is eliminated in older adults: age-related reduction in event-related brain potentials associated with evaluative categorization. Psychol Aging (2006) 21:815-20. doi:10.1037/0882-7974.21.4.815

47. Öhman A, Flykt A,Esteves F.Emotion drives attention: detecting the snakein the grass. J Exp Psychol Gen (2001) 130:466-78. doi:10.1037/0096-3445.130.3.466

Conflict of Interest Statement: The authors declare that the research was conducted in the absence of any commercial or financial relationships that could be construed as a potential conflict of interest.

Copyright $\odot 2017$ Suo, Liu, Chen and Zhang. This is an open-access article distributed under the terms of the Creative Commons Attribution License (CC BY). The use, distribution or reproduction in other forums is permitted, provided the original author(s) or licensor are credited and that the original publication in this journal is cited, in accordance with accepted academic practice. No use, distribution or reproduction is permitted which does not comply with these terms. 